\title{
Comparison of the value of novel rapid measurement of myoglobin, creatine kinase, and creatine kinase-MB with the electrocardiogram for the diagnosis of acute myocardial infarction
}

Hai Shiang Lee, Stephen J Cross, Paul Garthwaite, Arthur Dickie, Ian Ross, Stephen Walton, Kevin Jennings

\begin{abstract}
Objective-To determine whether serum myoglobin, creatine kinase, and creatine kinase-MB measured at admission by rapid, compact, and easy to use automated quantitative analysers (results within $10 \mathrm{~min}$ ) helped the early identification of acute myocardial infarction. The results were compared with the data obtained from the electrocardiograms recorded at admission.

Design-A prospective study.

Setting-Coronary care unit.

Patients-94 consecutive patients with suspected myocardial infarction. Myocardial infarction was subsequently confirmed in 44 patients and excluded in 50. Methods-All admission serum myoglobin, creatine kinase, and creatine kinase-MB were measured by clinical staff using analysers in the coronary care unit. An admission electrocardiogram was obtained from all patients.
\end{abstract}

Results-The sensitivity, specificity, and predictive accuracy for diagnosing myocardial infarction were: electrocardiogram $68 \%, 100 \%$, and $85 \%$; myoglobin $57 \%, 100 \%$, and $80 \%$; creatine kinase (threshold of $190 \mathrm{U} / 1$ ) $34 \%, 98 \%$, and 68\%; creatine kinase-MB (threshold of $25 \mathrm{U} / \mathbf{1}$ ) $43 \%, 100 \%$, and $73 \%$. When the electrocardiographic and myoglobin data were combined the sensitivity improved to $91 \%$, diagnostic accuracy to $96 \%$, with specificity of $100 \%$. The results for the electrocardiogram and creatine kinaseMB were $80 \%, 90 \%, 100 \%$ respectively and those for the electrocardiogram with creatine kinase were $80 \%, 89 \%, 98 \%$ respectively.

Conclusions-Admission myoglobin, creatine kinase, and creatine kinase-MB measurements were not as useful as the electrocardiogram for the diagnosis of acute myocardial infarction. Combining the electrocardiogram and myoglobin data substantially improved the sensitivity and predictive accuracy for the diagnosis of acute myocardial infarction.

(Br Heart f 1994;71:311-315)

Earlier biochemical identification of patients with acute myocardial infarction is desirable. These patients may benefit from early applica- tion of interventions such as thrombolysis. ${ }^{1}$ Though the electrocardiogram is widely used for diagnosing acute myocardial infarction its sensitivity can be as low as $60 \% .^{23}$

Myoglobin, creatine kinase (CK), and creatine kinase-MB (CK-MB) are released early after myocardial infarction..$^{4-8}$ Until recently, they could only be estimated either semi-quantitatively ${ }^{9}$ or by time consuming laboratory based procedures. ${ }^{1011}$ This made such estimation unsuitable for the early diagnosis of myocardial infarction. ${ }^{12}$ Novel rapid analysers for measuring myoglobin $\mathrm{CK}$, and CK-MB are now available. These give a quantitative result within 10 minutes of blood collection. The analyser is compact and can be placed in the coronary care unit and be used by clinical staff. This has made the early biochemical identification of infarct patients feasible.

We used the rapid analyser in a prospective trial to evaluate the potential of rapid myoglobin, CK, and CK-MB analysis in the early identification of acute myocardial infarction to see whether this approach gave useful information in addition to that already available from the presenting electrocardiogram.

\section{Patients and methods}

PATIENTS

We studied 94 consecutive patients who were admitted to the coronary care unit with chest pain and suspected myocardial infarction. We excluded patients who had had recent intramuscular injections.

Six patients were not included because the coronary care unit was busy and two more refused to participate. Myocardial infarction was diagnosed by the World Health Organisation criteria. ${ }^{13}$

\section{DATA COLLECTION}

Blood samples were collected at admission into a plain tube. They were left to stand for a minute and then centrifuged at $2500 \mathrm{rpm}$ for 4 min to obtain the serum used for analysis. Myoglobin, CK, and CK-MB were measured by one operator using analysers within the coronary care unit. Myoglobin, CK, and CKMB was measured either immediately after the patients were admitted or at least twice daily. Serum samples were stored at $4^{\circ} \mathrm{C}$ if the assay was not performed immediately. Electrocardiograms were recorded on admission and on days 2 and 3. Blood samples 
taken at admission and on days 2 and 3 were also sent to the laboratory for CK-MB analysis.

MYOGLOBIN ANALYSIS

We used a Behring Turbitimer system (Behringwerke Diagnostica), which is a particle-enhanced assay based on the reaction between myoglobin present in human serum or plasma and anti-myoglobin antibodies coupled to polystyrene particles. Turbidity is measured photometrically as the maximum reaction velocity ( $V \max$ ) of precipitate formation and the time required to attain Vmax. This method permits measurement on both sides of the Heidelberger-Kendall curve. The analyser (420 mm (length) $\times 325 \mathrm{~mm}$ (width) $\times 200 \mathrm{~mm}$ (height)) and printer $(420 \mathrm{~mm} \times$ $160 \mathrm{~mm} \times 100 \mathrm{~mm}$ respectively) are compact. We pipetted $50 \mu \mathrm{l}$ of serum into a cuvette and added $0.5 \mathrm{ml}$ of reagent. The stability of the analyser (under the supervision of the biochemists) was checked twice a week with control reagents. The analyser gives a quantitative result in about a minute from the time the serum sample is taken (range $50-650 \mu \mathrm{g} / \mathrm{l}$ ).

CK AND CK-MB ANALYSIS

We measured CK and CK-MB enzymes on a desk top Kodak Ektachem DT analyser (Eastman Kodak) which consists of two units $340 \mathrm{~mm}$ (width) $\times 340 \mathrm{~mm}$ (depth) $\times$ $170 \mathrm{~mm}$ (height) and $460 \mathrm{~mm} \times 340 \mathrm{~mm} \times$ $170 \mathrm{~mm}$ respectively. The reagents are individually packed as slides $(28 \mathrm{~mm} \times 28 \mathrm{~mm}$ $\times 1 \mathrm{~mm}$ ). The goat anti-human $M$ antibodies on the CK-MB slides inhibit the $M$ subunits of CK-MM and CK-MB enzymes. Residual $B$ activity is assayed. We used an automated electronic pipette to add $10 \mu \mathrm{l}$ of test serum to the slide. This gave a quantitative result (CK range $20-1600 \mathrm{U} / 1$ and $\mathrm{CK}-\mathrm{MB}$ of above 1-300 U/l) within $5 \mathrm{~min}$. The stability of the analyser was checked twice a week.

\section{DATA ANALYSIS}

Acute myocardial infarction was diagnosed if the patient had characteristic chest pain lasting for more than $30 \mathrm{~min}$; a rise of serum laboratory $\mathrm{CK}-\mathrm{MB}$ to at least twice the value of the upper limit of normal in our laboratory with a characteristic rise and fall of the enzyme; and ST elevation of $\geqslant 1 \mathrm{~mm}$ in two limb leads or $\geqslant 2 \mathrm{~mm}$ in two precordial leads.

For the purpose of calculation, myoglobin readings below the range of the analyser $(50 \mu \mathrm{g} / \mathrm{l})$ were taken as $49 \mu \mathrm{g} / 1$ and those readings above the range $(650 \mu \mathrm{g} / \mathrm{l})$ were taken as $651 \mu \mathrm{g} / \mathrm{l}$. We used the Wilcoxon unpaired rank sum test to compare the differences between group means.

\section{Results}

\section{STUDY POPULATION}

The patients (table 1) were admitted at a mean (SD) of $3.4(1.8) \mathrm{h}$ from the onset of symptoms. Myocardial infarction was confirmed in 44 patients. Among those with infarction 30 had ST elevation (16 inferior, 13 anterior, and one lateral), nine patients had
Table 1 Characteristics (mean(SD)) of patients

\begin{tabular}{lcl}
\hline Characteristic & $M I$ & No MI \\
\hline $\mathrm{n}$ & 44 & 50 \\
Age (y) & $58(8)$ & $53(9)$ \\
Male & 35 & 34 \\
Duration of chest pain (h) & $3 \cdot 2(1 \cdot 9)$ & $3 \cdot 5(1 \cdot 7)$ \\
Previous MI & $10(22 \cdot 7 \%)$ & $8(16 \%)$ \\
Myoglobin ( $\mu \mathrm{g} / \mathrm{l})$ & $219(185)$ & $68(17)$ \\
CK (U/1) & $321(450)$ & $81(66)$ \\
CK-MB (U/l) & $37(46)$ & $7(5)$ \\
\hline
\end{tabular}

MI, myocardial infarction; no MI, myocardial infarction excluded.

ST depression (seven anterolateral and two inferior), four patients had $\mathrm{T}$ wave inversion (three anterior and one inferior), and one had left bundle branch block on the presenting electrocardiogram. Of those patients in whom myocardial infarction was excluded 27 had normal electrocardiograms, 10 had $T$ wave inversion, four had abnormal $Q$ waves, two had abnormal $Q$ waves and ST depression, three had ST depression, three had atrial fibrillation, and one had bradycardia on their presenting electrocardiograms.

Fifty nine patients were not given thrombolysis. Myocardial infarction was later confirmed in 14 of these patients. Eight of these patients with myocardial infarction did not have ST elevation. In the six patients with ST elevation thrombolysis was contraindicated in five and the electrocardiogram was incorrectly interpreted in one.

Five patients in whom myocardial infarction was excluded were treated with thrombolysis because their history had suggested infarction. The presenting electrocardiogram was normal in three of these patients: the other two had anterolateral $\mathrm{T}$ wave inversion.

\section{ELECTROCARDIOGRAM}

Only $30(68 \%)$ of the 44 patients with confirmed myocardial infarction had the correct diagnosis made on the presenting electrocardiogram. None of the patients in whom infarction was excluded had ST elevation. This resulted in a specificity of $100 \%$ and predictive accuracy of $85 \%$ for the presenting electrocardiogram.

\section{MYOGLOBIN}

The mean (SD) myoglobin measurement of the patients with myocardial infarction (219 (185) $\mu \mathrm{g} / \mathrm{l}$ ) was significantly higher than the values for those patients in whom infarction was excluded (68 (17)), $\mathrm{p}<0.01)$. With the manufacturer's recommended threshold of $90 \mu \mathrm{g} / 1$ the sensitivity for diagnosing acute myocardial infarction was $75 \%$ (33 of 44 patients), the specificity was $92 \%$ (46 of 50 patients), and the diagnostic accuracy was $84 \%$.

However the highest myoglobin measurement for our series of patients without infarcts was $112 \mu \mathrm{g} / 1$. We therefore also determined the diagnostic yield at a myoglobin threshold of $120 \mu \mathrm{g} / 1$ when acute myocardial infarction was correctly identified in 25 of the 44 patients. This gave a sensitivity of $57 \%$, a specificity of $100 \%$, and a predictive accuracy 
Table 2 Sensitivity, specificity, and predictive accuracy (PA) of diagnosing acute myocardial infarction at myoglobin thresholds of 90,100,110, and $120 \mu \mathrm{g} / \mathrm{l}$

\begin{tabular}{llllll}
\hline \multicolumn{7}{l}{ Threshold $(\mu g / l)$} \\
\cline { 3 - 6 } & & 90 & 100 & 110 & 120 \\
& $n$ & $(n(\%))$ & $(n(\%))$ & $(n(\%))$ & $(n(\%))$ \\
\hline Sensitivity & $44^{\star}$ & $33(75)$ & $30(68)$ & $29(66)$ & $25(57)$ \\
Specificity & $50 \dagger$ & $46(92)$ & $49(98)$ & $49(98)$ & $50(100)$ \\
PA & $94 \ddagger$ & $79(84)$ & $79(84)$ & $78(83)$ & $75(80)$ \\
\hline
\end{tabular}

* Number of patients with myocardial infarction, $\nmid$ number of patients in whom myocardial infarction was subsequently excluded, $\ddagger$ all patients.

- Non-MI O MI with ST elevation $\bullet$ MI with no ST elevation

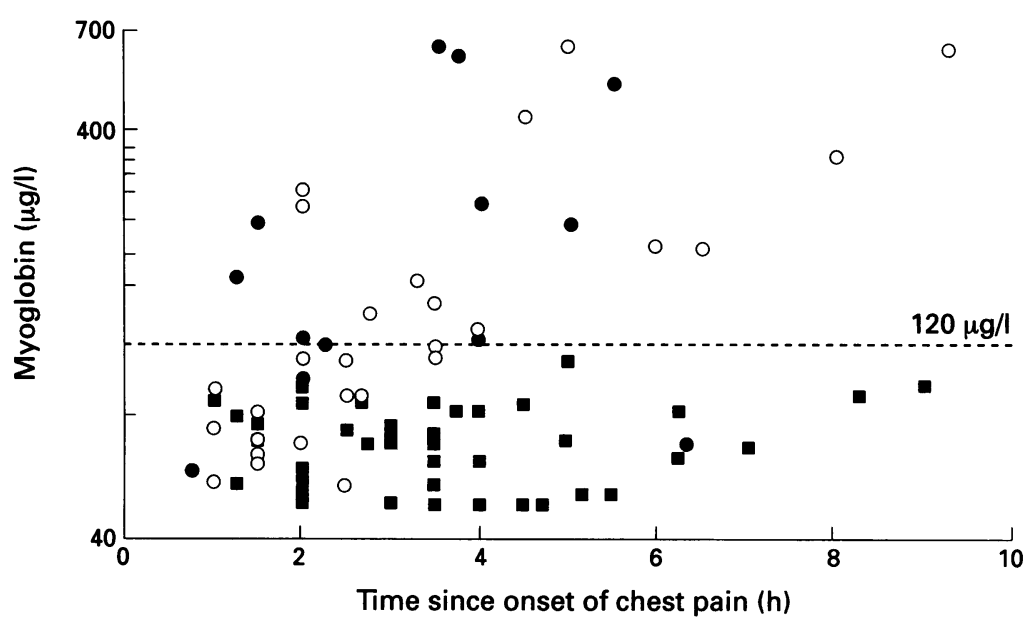

Figure 1 Myoglobin concentration plotted against time since onset of chest pain. Non$M I$, patients in whom myocardial infarction was subsequently excluded; MI with ST elevation, infarct patients with ST elevation in the presenting electrocardiogram; MI with no ST elevation, Infarct patients with no ST elevation in the presenting electrocardiogram.

of $80 \%$. Table 2 summarises the diagnostic yield obtained when we used other myoglobin thresholds. Figure 1 shows the myoglobin measurement for all patients and for patients with myocardial infarction with and without ST elevation on their presenting electrocardiogram. Myoglobin measurements were $\geqslant 120 \mu \mathrm{g} / 1$ in 10 infarct patients without ST elevation on their presenting electrocardiogram.

MYOGLOBIN AND THE ELECTROCARDIOGRAM

The myoglobin measurement at admission gave a lower diagnostic yield than the electrocardiogram. However, even when we used a higher threshold $(120 \mu \mathrm{g} / \mathrm{l})$ than that recommended by the manufacturer myoglobin mea-

Table 3 Sensitivity, specificity, and predictive accuracy (PA) of diagnosing acute myocardial infarction at creatine kinase thresholds of 100,150,170, and $190 \mathrm{U} / \mathrm{l}$

\begin{tabular}{llllll}
\hline \multicolumn{7}{c}{ Threshold (UIl) } \\
\cline { 3 - 6 } & $n$ & 100 & 150 & 170 & 190 \\
& $n$ & $(n(\%))$ & $(n(\%))$ & $(n(\%))$ & $(n(\%))$ \\
\hline Sensitivity & $44^{\star}$ & $31(70)$ & $20(45)$ & $18(41)$ & $15(34)$ \\
Specificity & $50 \dagger$ & $32(64)$ & $44(88)$ & $46(92)$ & $49(98)$ \\
PA & $94 \ddagger$ & $63(67)$ & $64(68)$ & $64(68)$ & $64(68)$ \\
\hline
\end{tabular}

^ Number of patients with myocardial infarction, $†$ number of patients in whom myocardial infarction was subsequently excluded; $¥$ all patients. surements still identified 10 further infarct patients without ST elevation on their presenting electrocardiogram. When we used both indices we increased the sensitivity to $91 \%$ and the predictive accuracy to $96 \%$ (specificity $100 \%$ ).

\section{CREATINE KINASE}

The mean (SD) CK measurement of the infarct patients (321 (450) U/l) was significantly $(\mathrm{p}<0.01)$ higher than that in patients in whom infarction was subsequently excluded (81 (66) $U / 1)$. The manufacturer recommended that a CK measurement of $<65 \mathrm{U} / 1$ should be regarded as negative. When we used this threshold the sensitivity for diagnosing myocardial infarction was $84 \%$ (37 of 44 patients), specificity was $50 \%$ (25 of 50 patients) and the diagnostic accuracy was $66 \%$.

All but one of the patients without myocardial infarction had a CK reading of $<190$ $\mathrm{U} / 1$. This threshold correctly identified acute myocardial infarction in only 15 of the 44 patients. Five infarct patients without ST elevation on their presenting electrocardiogram and one patient in whom infarction was excluded had a CK measurement of $>190$ $\mathrm{U} / 1$. This gave a sensitivity of $34 \%$, a specificity of $98 \%$, and a predictive accuracy of $68 \%$. Table 3 summarises the diagnostic yield with other $\mathrm{CK}$ thresholds and Fig 2 shows the plot of CK readings for all patients.

\section{CREATINE KINASE-MB}

Mean CK-MB in the infarct patients (37 (46) $\mathrm{U} / \mathrm{l}$ ) was significantly higher than in those patients in whom infarction was subsequently excluded (mean 7(5) U/l, p < 0.01). The manufacturer recommends that the CK-MB must be $>16 \mathrm{U} / 1$ and between $4-25 \%$ of the total CK to be diagnostic of acute myocardial infarction. When we used this threshold the sensitivity was $50 \%$ ( 22 of 44 patients), the specificity was $92 \%$ (46 of 50 patients), and the diagnostic accuracy was $72 \%$.

In none of the patients without infarcts was the $\mathrm{CK}-\mathrm{MB}$ value $>25 \mathrm{U} / \mathrm{l}$. At this threshold, acute myocardial infarction was correctly identified in 19 of the 44 patients by the CK$M B$ reading at admission. Five infarct patients without ST elevation in their presenting electrocardiogram had a $\mathrm{CK}-\mathrm{MB}$ reading of $>25 \mathrm{U} / 1$ whereas none of the patients in whom infarction was subsequently excluded did. This gave a sensitivity of $43 \%$, a specificity of $100 \%$, and predictive accuracy of $73 \%$. Table 4 summarises the diagnostic yield using other $\mathrm{CK}-\mathrm{MB}$ thresholds and figure 3 shows the CK-MB readings for all patients.

CK, CK-MB, AND THE ELECTROCARDIOGRAM The diagnostic yield of admission $C K$ and CK-MB readings was lower than that of the electrocardiogram. Both enzymes readings, however, identified five further infarct patients without ST elevation in their presenting electrocardiogram. A combination of the electrocardiographic and CK-MB data (threshold $25 \mathrm{U} / 1$ ) gave a higher sensitivity of 


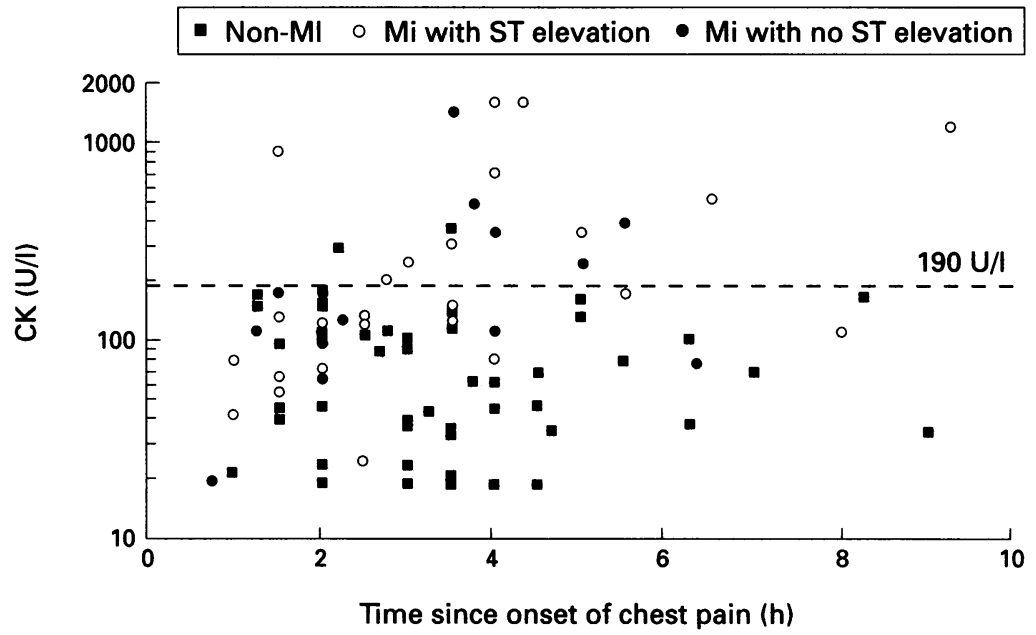

Figure 2 Creatine kinase readings plotted against time since onset of chest pain. See legend to fig 1 for description of subgroups.

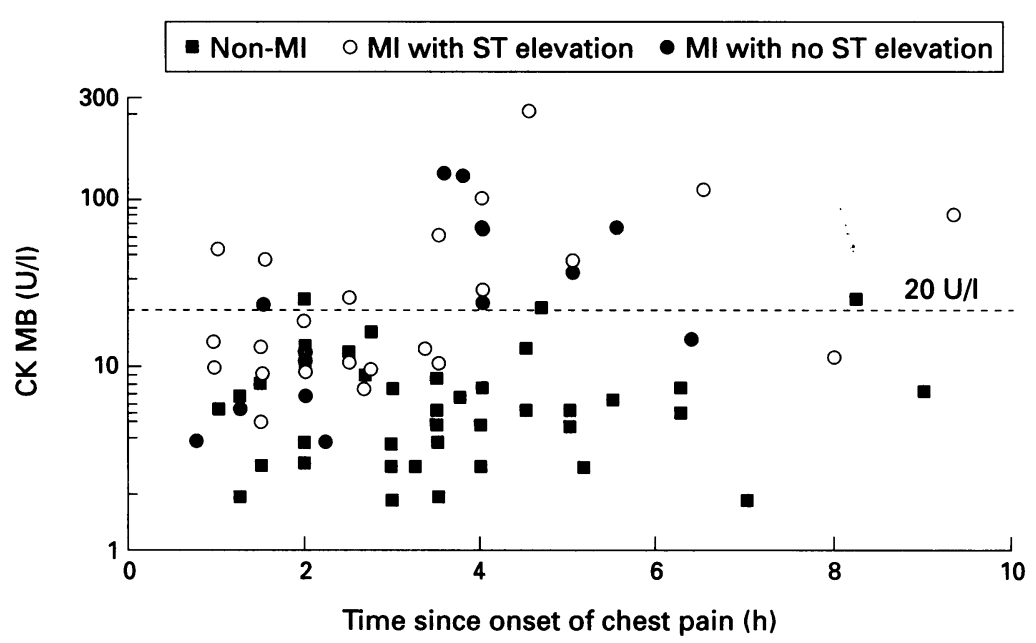

Figure 3 Creatine kinase-MB readings plotted against time since onset of chest pain. See legend to fig 1 for description of subgroups.

$80 \%$. The specificity and diagnostic accuracy were $100 \%$ and $90 \%$. For a combination of CK (threshold $190 \mathrm{U} / \mathrm{l}$ ) and electrocardiographic data the sensitivity was $80 \%$, specificity $98 \%$, and diagnostic accuracy $89 \%$.

\section{Discussion}

These results can be available by the time the clinical details were obtained from the

Table 4 Sensitivity, specificity, and predictive accuracy $(P A)$ of diagnosing acute myocardial infarction at creatine kinase-MB thresholds of 15,20 , and $25 \mathrm{U} / \mathrm{l}$

\begin{tabular}{lllll}
\hline \multicolumn{5}{l}{ Threshold (U/l) } \\
\cline { 3 - 5 } & & 15 & 20 & 25 \\
& $n$ & $(n(\%))$ & $(n(\%))$ & $(n(\%))$ \\
\hline Sensitivity & $44^{\star}$ & $23(52)$ & $21(48)$ & $17(39)$ \\
Specificity & $50 \dagger$ & $46(92)$ & $47(94)$ & $50(100)$ \\
PA & $94 \ddagger$ & $69(73)$ & $68(72)$ & $67(71)$ \\
\hline
\end{tabular}

* Number of patients with myocardial infarction, $\nmid$ number of patients in whom myocardial infarction was subsequently excluded, $\ddagger$ all patients. patients and the clinical examination performed. This system of analysis would aid the management of patients rather than merely establish a diagnosis of myocardial infarction after management decision had been made. In addition the availability of a quantitative result (rather than a semiquantitative result) will lead to a diagnostic threshold for myocardial infarction.

Up to $40 \%$ of patients with acute myocardial infarction do not develop ST elevation on the admission electrocardiogram. ${ }^{23}$ The early identification of myocardial infarction in those patients without ST elevation may permit an increase in interventions aimed at reducing morbidity and mortality. ${ }^{1}$ Though these interventions may greatly benefit the patients with myocardial infarcts they are not without adverse effects. Thrombolysis, for example, can cause cerebral and gastrointestinal haemorrhage. To avoid exposing patients without myocardial infarction to this, we also examined the value of using a higher myoglobin, CK, and CK-MB thresholds than recommended by the manufacturer (to achieve higher specificity). We investigated whether the use of higher thresholds would still provide useful information in addition to that already available from the electrocardiogram. Using these thresholds, we found that admission myoglobin, $\mathrm{CK}$, and $\mathrm{CK}-\mathrm{MB}$ readings gave a lower diagnostic yield than the presenting electrocardiogram. When we used a combination of myoglobin and electrocardiographic data, however, the sensitivity improved from $68 \%$ with the electrocardiogram data and $57 \%$ with the myoglobin results alone to $91 \%$ with both sets of data. The predictive accuracy improved from $85 \%$ and $80 \%$ respectively to $96 \%$. Specificity was $100 \%$. To a lesser extent than the myoglobin data, the combination of electrocardiographic data and $\mathrm{CK}$ or $\mathrm{CK}-\mathrm{MB}$ readings also increased the sensitivity and diagnostic accuracy of either data set alone (CK to $80 \%$ and $89 \%$, CK-MB to $80 \%$ and $90 \%$ respectively). With CK, however, the specificity was lower (98\%).

Patients with myocardial infarction but no ST elevation may benefit from thrombolytic therapy. The control group of patients showing ST depression in the ISIS-2 (International Study of Infarct Survival) had a surprisingly high mortality (18.6\%) (105 vascular deaths/566 patients), which was higher than even the control groups of patients with anterior $(18.0 \%)$, inferior $(8.8 \%)$, and inferior plus anterior $(15 \cdot 8 \%)$ ST elevation. ${ }^{14}$ The GISSI trial showed similar findings. ${ }^{15}$ Thrombolytic therapy, however, did not reduce mortality in these patients. The reason for these findings is not clear. In these trials some patients with ST depression who were treated with thrombolysis were subsequently shown not to have had an infarct. It is likely that if thrombolytic therapy is given to all patients with ST depression the benefits conferred on those with myocardial infarction will be diluted by those without myocardial infarction and some of the patients without 
infarction but given thrombolysis will be exposed unnecessarily to their potential adverse effects. Thrombolytic therapy may, however, benefit the subset of patients with ST depression and myocardial infarction confirmed by early enzyme analysis. Future study will be needed to clarify this.

When myocardial infarction is complicated by bundle branch block it indicates more extensive myocardial damage. ${ }^{16}$ Early identification of acute myocardial infarction in these patients would be particularly helpful because they would benefit from thrombolysis. ${ }^{14}$

In patients with suspected myocardial infarction who presented with a normal electrocardiogram in the ISIS-2 trial mortality was low $(2 \cdot 6 \%){ }^{14}$ This mortality was not reduced by thrombolytic or aspirin therapy or both. Three of our patients with normal electrocardiogram were given thrombolytic therapy. In view of the potential adverse effect, thrombolysis should not be given to this group of patients.

The purpose of this study was to determine whether the myoglobin, $\mathrm{CK}$, and $\mathrm{CK}-\mathrm{MB}$ values at admission are helpful in the early identification of acute myocardial infarction. A low admission reading, however, does not exclude myocardial infarction or unstable angina and these patients must not be discharged from the hospital on the basis of these results alone. As with other investigations, early biochemical analysis should be used only as an aid to the management of the patients with suspected myocardial infarction.

Our results indicate that admission myoglobin, $\mathrm{CK}$, and $\mathrm{CK}-\mathrm{MB}$ have a lower sensitivity and diagnostic accuracy for the diagnosis of acute myocardial infarction than the admission electrocardiogram. However, myoglobin readings in particular provide valuable information in addition to that obtained from the presenting electrocardiogram. Combining the myoglobin and electrocardiographic data substantially increases the sensitivity and diagnostic accuracy provided by either value alone. Studies are needed to assess how this information can help the management of the coronary care unit and the administration of thrombolysis.

1 Lee TH, Weisberg MC, Brand DA, Rouan GW, Goldman L. Candidates for thrombolysis among emergency room patients with acute chest pain. Ann Intern Med 1989; 110:957-62.

2 Yusuf S, Pearson M, Sterry $H$, et al. The entry ECG in the early diagnosis and prognostic stratification of patients with acute myocardial infarction. Eur Heart $\mathcal{F} 1984 ; 5$ : with acute. 6 .

3 Rude RE, Poole WK, Muller JE, et al. Electrocardiographic and clinical criteria for recognition of cardiographic and clinical criteria for recognition of patients. Am 7 Cardiol 1983;52:936-42.

4 Ohman EM, Casey C, Bengtson JR, Pryor D, Tormey W, Horgan JH. Early detection of acute myocardial infarction: additional diagnostic information from serum concentrations of myoglobin in patients without ST elevation. Br Heart F 1990;63:335-8.

5 Drexel H, Dworzak E, Kirchmair W, Milz M, Puschendorf B, Dienstl F. Myoglobinaemia in the early phase of acute myocardial infarction. Am Heart $\mathcal{f} 1983 ; 105: 642-51$.

6 Marin MM, Teichman SL. Use of rapid serial sampling of creatine kinase $\mathrm{MB}$ for very early detection of myocardial infarction in patients with acute chest pain. $\mathrm{Am}$ Heart $\mathcal{1}$ 1992;123:354-61.

7 Sidney B, Rosalky J. Enzyme assays in diseases of the heart and skeletal muscle. 7 Clin Pathol 1970;24(suppl 4): 60-70.

8 Stone MJ, Willerson JT. Myoglobinaemia in myocardial infarction. Int $\mathcal{F}$ Cardiol 1983;4:49-52.

9 Konings CH, Funke Kupper AJ, Verheugt FW. Comparison of two latex agglutination test kits for serum myoglobin in the exclusion of acute myocardial infarction. Acta Clin Biochem 1989;26:254-8.

10 Vaananen HK, Syrjela H, Rahkila P, Vuori J, Melamies LM, Myllyla V, Takala TE. Serum carbonic anhydrase iii and myoglobin concentration in acute myocardial ii and myoglobin concentration in

11 Young P, Hedges J, Gibler W, et al. Do CK-MB results affect chest pain decision making in the emergency department? Ann Emerg Med 1991;20:1220-8.

12 Timmis AD. Early diagnosis of acute myocardial infarction. $B M \mathcal{F}$ 1990;301:941-2.

13 World Health Organisation criteria for the diagnosis of acute myocardial infarction. Proposed for the multinational monitoring of trends and determinant in cardiovascular disease. Geneva: WHO Cardiovascular Disease Unit, 1981.

14 ISIS-2 Collaborative group. Randomised trial of intravenous streptokinase, oral aspirin, both, or neither among 17187 cases of suspected acute myocardial infarction. Lancet 1988;ii:349-60.

15 Gruppo Italiano per lo studio della streptochinasi nell'infarto miocardico (GISSI). Effectiveness of intravenous thrombolytic treatment in acute myocardial infarction. Lancet 1986;1:397-402.

16 Pierard LA, Chapelle JP, Albert A, Dubois C, Kulbertus HE. Characteristics associated with early (less than or equal to 3 months) versus late (greater than 3 months to less than or equal to 3 years) mortality after acute myocardial infarction. Am f Cardiol 1989;64:315-8. 